\title{
CARACTERIZACIÓN BIOPSICOSOCIAL DE UNA POBLACIÓN DE ADOLESCENTES EMBARAZADAS \\ EXPERIENCIA EN UNA EMPRESA PROMOTORA DE SALUD, BOGOTÁ DC, COLOMBIA
}

Silvia Lorena Mora Villa-Roel MD*, Alexander Ocampo Serna MD**, Manuel Salvador Peña Castro MD**

\section{Resumen}

El embarazo en la adolescencia se reconoce en el mundo como un problema de salud pública de etiología multifactorial, generador de un mayor riesgo de complicaciones durante la gestación, el parto y el puerperio, que incrementa las tasas de morbilidad y mortalidad materna y perinatal. Objetivo: describir el perfil biopsicosocial y los resultados materno perinatales de una población de adolescentes embarazadas afiliadas a una empresa promotora de salud (EPS-S) en Bogotá DC en el primer semestre del año 2011. Métodos: estudio observacional descriptivo de una cohorte de 121 pacientes ingresadas en cualquier trimestre de la gestación y seguidas hasta el parto. Se describen variables sociofamiliares, demográficas y desenlaces maternos y perinatales. Resultados: edad promedio 17.3 años (DE 1.3), la tipología familiar más común fue nuclear $57 \%$ y extensa $20.6 \%$ con disfunción familiar en $38.9 \%$. El $63.6 \%$ tenía antecedente familiar de embarazo en adolescentes, siendo la madre la más frecuente $68.8 \%$. El 75.2\% no planificaba y $64.5 \%$ no deseaba la gestación. Las comorbilidades en el curso del embarazo fueron infección de vías urinarias $27.36 \%$ y vaginosis $24 \%$. Se presentó distocia del descenso en $9.1 \%$, lesiones del canal $4.1 \%$, trastornos hipertensivos $4.1 \%$ y bajo peso al nacer $4.9 \%$. La mortalidad neonatal fue $1.6 \%$, no se reportó mortalidad materna. Conclusión: no se observó mayor frecuencia de complicaciones durante la gestación ni en el parto respecto a otras series de gestantes no adolescentes, la mortalidad neonatal es igual a la observada para todas las edades a nivel local y nacional. Aunque se ha propuesto que este grupo es de alto riesgo es probable que al no tener población en adolescencia temprana se minimizan las complicaciones. No se puede desconocer el riesgo social derivado de la disfunción familiar.

Palabras clave: adolescente, embarazo, familia, complicaciones, psicosocial.

\section{CHARACTERIZATION OF THE BEHAVIORAL AND SOCIO-DEMOGRAPHIC PROFILE OF A POPULATION OF PREGNANT ADOLESCENTS}

\section{Abstract}

Teen pregnancy is globally recognized as a public health problem of multifactorial etiology. Pregnant teens have a greater risk of pregnancy, birth and puerperium complications thus increasing maternal and perinatal morbidity and mortality. Objective: To describe the behavioral and socio-demographic profile and perinatal maternal outcomes of a population of pregnant teens affiliated to a Health Maintenance Organization (HMO) in Bogotá, during the first semester of 2011. Methods: this is a descriptive observational cohort study including 121 pregnant adolescents recruited at any gestational age, followed up until giving birth. Social, familial and demographic variables as well as maternal

Fecha recibido: junio 29 de 2012 - Fecha aceptado: marzo 22 de 2013

* Especialista en Medicina Familiar. Subdirector Médico del Hospital de San José. Profesor Asociado, Fundación Universitaria de Ciencias de la Salud. Bogotá DC. Colombia.
** Residente III de Medicina Familiar, Fundación Universitaria de Ciencias de la Salud. Bogotá DC. Colombia. 
and perinatal outcomes were assessed. Results: Mean maternal age was 17.3 years (SD 1.3). Most patients (57\%) belonged to nuclear families and $20.6 \%$ to extended families; family dysfunction was identified in $38.9 \%$; $63.6 \%$ had a family history of teen pregnancies, most of them born from a teen mother themselves $(68.8 \%) ; 75.2 \%$ did not use any contraceptive method; and $\mathbf{6 4 . 5 \%}$ did not want their pregnancy. The most frequent comorbidities during pregnancy were urinary tract infection $(\mathbf{2 7 . 3 6 \%})$ and vaginosis $\mathbf{2 4 \%}$ ). Dystocia presented in $\mathbf{9 . 1 \%}$, birth injuries to the mother in $4.1 \%$, hypertensive disorders in $4.1 \%$, and a low birth-weight baby in $4.9 \%$. Neonatal mortality was $1.6 \%$. No maternal mortality was reported. Conclusion: A greater number of pregnancy or delivery complications were not evidenced when compared to other series of pregnancies in women older than 20 . Neonatal mortality is similar to that observed in all age groups at local and national levels. Although this group has been proposed as a high risk group, probably not having an early adolescence population minimizes complications. Social risk derived from family dysfunction should not be disregarded.

Key words: adolescent, pregnancy, family, complications, socio-demographic.

\section{Introducción}

El embarazo en la adolescente se considera un problema de salud pública que genera un incremento en el riesgo de morbimortalidad materna y perinatal, por las diferentes complicaciones en el transcurso del embarazo y los resultados finales de la madre y su hijo, como son nacimientos pretérmino y bajo peso al nacer, sin dejar de lado las secuelas de índole psicosocial como la deserción escolar, pobreza, maltrato, violencia y abuso sexual. Elementos de índole biológicos, psicológicos y socioculturales establecen el carácter multifactorial del problema. ${ }^{1-4}$

La sociedad actual discrimina y resta oportunidades a las adolescentes embarazadas, exigiéndoles un cambio del rol en la comunidad; es lamentable cómo existe una perpetuación de esta condición que se refleja en las hijas, pasando la misma situación de generación en generación. ${ }^{3,4}$ Además, se conoce la existencia de un alto porcentaje de embarazos no deseados en este grupo etario; estudios en países en vía de desarrollo reportan de $20 \%$ a $60 \% .^{3,5,6}$

Los países desarrollados no son ajenos a esta problemática. Es así como en Estados Unidos se presenta una tasa de 41.2 nacidos por cada 1.000 adolescentes (2004), con cifras que duplican a Gran Bretaña, Canadá y son casi cuatro veces mayores que Francia y Suecia. Dos tercios de estos son en adolescentes tardías ${ }^{3}$, mujeres entre los 18 y 19 años, que en países como Colombia han alcanzado la mayoría de edad. ${ }^{3,5,6}$
A nivel local, en Cundinamarca de 34.128 nacidos vivos el $24.1 \%$, es decir 8.944 son hijos de mujeres menores de 19 años y de esta cifra $1 \%$ con menos de 15 años. En 2008 en Bogotá se presentaron 573 nacimientos en adolescentes entre los 10 y los 14 años, y 20.709 nacimientos en el rango de edad de 15 a 19, siendo las localidades de Ciudad Bolívar, Kennedy, Bosa, Suba y San Cristóbal las de mayor número de embarazos. ${ }^{6-8}$

Es difícil establecer cuál de todos los factores demográficos, clínicos o familiares explican el incremento de embarazos en adolescentes; lo que si se ha visto es que las madres de escasos recursos económicos asumen el papel de proveedoras de los hogares generando cambios significativos en la dinámica familiar, porque han tenido que dejar los roles en su función de administradoras del hogar y como consecuencia las jóvenes carecen de modelos morales de referencia para guiar sus conductas y muchas de las funciones de los adultos se transfieren temprano a las adolescentes. ${ }^{9,10}$ La caracterización del comportamiento de estas últimas va a depender de múltiples variables demográficas, ambientales, culturales, familiares, raciales, ecológicas, estrato socioeconómico, origen étnico y período de la adolescencia, entre otras. ${ }^{11-15}$

Las tareas que deben realizar las adolescentes son muy complejas, entre ellas podemos nombrar identificación de su proyecto de vida, emancipación de la familia de origen, dirigir y controlar su conducta de acuerdo con su propia escala de valores y definir su identidad sexual, características que desea en su futura pareja y 
una de las más importantes que es establecer su identidad propia. Si todo lo anterior se suma a la presencia de un embarazo deseado o no, podría comprometer el cumplimiento de los compromisos propios de su ciclo vital individual. ${ }^{12-16}$

El mayor conocimiento de nuestra población de adolescentes gestantes facilitaría identificar puntos fundamentales de intervención temprana, en determinantes no solo demográficos y clínicos sino los de la esfera familiar. El objetivo de este estudio es describir el perfil biopsicosocial y los resultados maternos y perinatales en la población de adolescentes embarazadas de una empresa promotora de salud (EPS-S) en la ciudad de Bogotá DC que acuden a un programa de control prenatal.

\section{Materiales y métodos}

Estudio observacional descriptivo de una cohorte de adolescentes gestantes que asistieron a la consulta de control prenatal en el primer semestre del año 2011. Ingresaron aquellas de 11 a 19 años de edad embarazadas independiente de su edad gestacional. Se reclutaron casos del 2 de abril al 30 de septiembre de 2011 y se hizo seguimiento hasta la terminación del embarazo. Las que no residían en la ciudad de Bogotá DC y que no asistieron al curso psicoprofiláctico fueron excluidas. La política de demanda inducida por la EPS hacia las gestantes asegura una inclusión en el programa de control prenatal del $88.7 \%$.

La recolección de datos se realizó mediante encuesta directa con la paciente los días de asistencia al curso psicoprofiláctico o durante el desarrollo de la jornada de promoción y prevención del programa de maternidad segura, a la cual fueron invitadas todas las adolescentes gestantes de la ciudad de Bogotá DC registradas en las bases de datos de la IPS. Se programaron visitas a las unidades de atención básica en cinco diferentes puntos de la ciudad para identificar otras gestantes que cumplieran criterios de inclusión. Tanto la encuesta como la aplicación de instrumentos de medicina familiar las realizaron especialistas en formación capacitados para la realización e interpretación de los mismos, previo diligenciamiento del consenti- miento informado por la paciente y el acudiente para las menores de 18 años. Se aplicó la encuesta con cinco secciones definidas: identificación, evaluación familiar, antecedentes ginecobstétricos, características del embarazo actual y terminación del embarazo. Se estableció el APGAR familiar, clasificando la funcionalidad familiar así: normal 18 a 20 puntos, disfunción leve de 14 a 17 , moderada de 10 a 13 y severa $<9$ puntos ${ }^{17}$, y el familiograma, con tres líneas generacionales. Se definió adolescencia temprana cuando la edad fue de 10 a 13 años y 11 meses, media entre 14 y 16 años y 11 meses, y adolescencia tardía de 17 a 19 años y 11 meses. ${ }^{17,18}$

Además del protocolo dentro del programa de control prenatal de la IPS se hizo seguimiento telefónico de cada caso y mediante comunicación directa se obtuvo información acerca del desenlace de la gestación y las complicaciones maternas y perinatales. Todos los datos fueron verificados con el sistema de información mediante registro electrónico de la EPS. Se anotaron las complicaciones maternas relacionadas con hemorragia posparto, trastornos hipertensivos del embarazo, alteraciones del canal del parto, distocia en el descenso y la dilatación. En el recién nacido se reportaron bajo peso al nacer, aspiración de meconio, sepsis, macrosomía y malformaciones.

Mediante estadística descriptiva se resumen las variables cuantitativas como promedios y desviaciones estándar, rangos. Las variables cualitativas se presentancomo frecuencias absolutas y relativas. En el análisis de correspondencias múltiples se definieron como variables ilustrativas las complicaciones en el curso de la gestación y las que ocurrieron en el desenlace de la gestación, y como variables activas las características demográficas, familiares y clínicas. El análisis se realizó con el programa Stata 10.0 y Spad 7.

El protocolo fue evaluado y aprobado por el comité de investigaciones y ética de la facultad de medicina de la Fundación Universitaria de Ciencias de la Salud, el comité de ética en investigaciones con seres humanos del Hospital de San José de Bogotá DC y por el comité de ética e investigaciones de la IPS. 


\section{Resultados}

En la base de datos de la EPS se identificaron 42.727 mujeres dentro de la edad de interés, 490 con diagnóstico de embarazo confirmado, de las cuales cumplieron criterios de inclusión 141 hasta el final del período de reclutamiento. Al terminar el seguimiento y la evaluación de desenlaces de los casos, se cierra el estudio con 121 pacientes.

Las características demográficas se presentan en la Tabla 1. Se encontró una edad promedio de 17.3 años (DE 1.3 años), rango 14-19 años, es decir no se identificaron

\section{Tabla I. Características demográficas}

\begin{tabular}{|c|c|c|}
\hline Edad, promedio (DE) & 17.3 & $(1.3)$ \\
\hline \multicolumn{3}{|c|}{ Nivel socioeconómico, frecuencia (\%) $\Omega$} \\
\hline II & 77 & $(63.6)$ \\
\hline I & 23 & $(19.0)$ \\
\hline III & 16 & $(13.2)$ \\
\hline no sabe/no responde & 5 & $(4.1)$ \\
\hline IV-V-VI & 0 & $(0)$ \\
\hline \multicolumn{3}{|c|}{ Ocupación, frecuencia (\%) } \\
\hline estudiante & 66 & $(54.5)$ \\
\hline ama de casa & 42 & $(34.7)$ \\
\hline empleada & 11 & $(9.1)$ \\
\hline otra & 2 & $(1.6)$ \\
\hline
\end{tabular}

Tipo de afiliación, frecuencia (\%)

\begin{tabular}{|l|l|l|}
\hline beneficiaria & 98 & $(80.9)$ \\
\hline afiliada & 23 & $(19.0)$ \\
\hline
\end{tabular}

\begin{tabular}{|l|c|c|}
\hline Localidad, frecuencia (\%) & \multicolumn{2}{|c|}{} \\
\hline Bosa & 24 & $(20.7)$ \\
\hline Ciudad Bolivar & 18 & $(15.5)$ \\
\hline Kennedy & 17 & $(14.6)$ \\
\hline Rafael Uribe Uribe & 12 & $(10.3)$ \\
\hline no sabe & 11 & $(9.5)$ \\
\hline San Cristóbal & 10 & $(8.6)$ \\
\hline Usme & 9 & $(7.7)$ \\
\hline Tunjuelito & 7 & $(6.0)$ \\
\hline Suba & 3 & $(2.6)$ \\
\hline Puente Aranda & 2 & $(1.7)$ \\
\hline
\end{tabular}

casos en la adolescencia temprana. En cuanto a nivel socioeconómico, en estrato uno hubo 23 (19\%) y en el dos 77 pacientes $(63.6 \%)$, procedentes del sector suroccidental de la ciudad $59(50.8 \%)$ y conviviendo con su pareja $69(57 \%)$. La mayoría cursaban estudios de secundaria $95(78.5 \%)$ y $19(15.7 \%)$ estaban en educación superior. Dedicadas a actividades del hogar $42(34.7 \%)$ y trabajaban solo $11(9.1 \%)$.

En cuanto a las características familiares, para tipología encontramos predominio nuclear $69(57 \%)$ y familia extensa $25(20.6 \%)$. De igual forma $61(51.3 \%)$ con ciclo vital en expansión y en apertura 32 (26.4\%), en

\begin{tabular}{|l|c|c|}
\hline Barrios Unidos & I & $(0.8)$ \\
\hline Fontibón & I & $(0.8)$ \\
\hline Usaquén & I & $(0.8)$ \\
\hline Teusaquillo & 0 & $(0)$ \\
\hline Chapinero & 0 & $(0)$ \\
\hline Antonio Nariño & 0 & $(0)$ \\
\hline Los Mártires & 0 & $(0)$ \\
\hline Santa Fe & 0 & $(0)$ \\
\hline Engativá & 0 & $(0)$ \\
\hline La Candelaria & 0 & $(0)$ \\
\hline Sumpaz & 0 & $(0)$ \\
\hline no aplica & 5 & \\
\hline Escolaridad & \multicolumn{2}{|c|}{} \\
\hline secundaria & 95 & $(78.5)$ \\
\hline técnico & 14 & $(11.6)$ \\
\hline primaria & 7 & $(5.8)$ \\
\hline profesional & 5 & $(4.1)$ \\
\hline ninguno & 0 & $(0)$ \\
\hline Estado civil & \multicolumn{2}{|c|}{} \\
\hline unión libre & 67 & $(55.4)$ \\
\hline soltera & 40 & $(40.5)$ \\
\hline otro & 3 & $(2.5)$ \\
\hline casada & 2 & $(1.6)$ \\
\hline separada & 0 & $(0)$ \\
\hline viuda & 0 & $(0)$ \\
\hline Procedencia & 118 & $(97.5)$ \\
\hline urbana & 3 & $(2.5)$ \\
\hline rural & \multicolumn{2}{|c|}{} \\
\hline & 0 \\
\hline
\end{tabular}

$\Omega$. Definido por planeación nacional documentado en recibo de servicios públicos, auto-referido; procedente de área metropolitana, fuera de Bogotá; a. nivel alcanzado en el momento de la entrevista. 
muchos casos determinadas por la misma paciente. $\mathrm{Al}$ evaluar la funcionalidad, encontramos $74(61.1 \%)$ de tipo normal, 7 (5.8\%) con disfunción familiar severa y $11(9.1 \%)$ moderada (Tabla 2 ).

Al evaluar el estado civil de los padres, encontramos unión libre 40 (33\%) y separados 44 (36.3\%), para el antecedente de embarazo adolescente en otra familiar $77(63.6 \%)$ en la mayoría de los casos fue la madre 53 $(68.8 \%)$, con una edad promedio de primer embarazo para el familiar con antecedente de embarazo adolescente de 16.8 años (DE 1.2).

El promedio de edad para el inicio de relaciones sexuales fue 15.6 años (DE 1.4), 71 (58.7\%) referían haber tenido un solo compañero sexual, 41 (33.9\%) entre dos y tres y $9(7.4 \%)$ cuatro o más hasta el momento de embarazarse, $102(84.3 \%)$ eran primigestantes, 18 (14.9\%) era su segundo embarazo y para una $(0.83 \%)$ el tercero, adolescente de 17 años con una sexarquia a los 14 años, uno de los productos fue un aborto y en el presente embarazo presentó como complicación materna hemorragia posparto. No planificaban 91 (75.2\%) y nunca habían asistido a un programa de planificación familiar 101(83.5\%) y de ellas $78(64.5 \%)$ no deseaban la gestación (Tabla 3).

Para iniciar el control prenatal 69 adolescentes (57\%) lo hicieron en el primer trimestre, 48 (39.7\%) en el segundo y 4 (3.3\%) en el tercero. Al momento de la encuesta reportaron suplencia con micronutrientes 117 pacientes (96.7\%), tenían vacuna de Td 93 (76.9\%) y valoración odontológica 85 (70.3\%) (Tabla 4).

En los estudios de tamizaje para las gestantes las alteraciones de la glicemia se presentaron en tres casos (2.5\%): dos por debajo de $60 \mathrm{mg} / \mathrm{dl}$ y un caso en 107 $\mathrm{mg} / \mathrm{dl}$, toxoplasma IgG positivo en $7.4 \%$, parcial de orina anormal 29 (23.9\%), urocultivo patológico 28 (23.1\%) y con ecografía anormal $7.4 \%$, dentro de esta última se incluyeron como patológicos la restricción del crecimiento intrauterino, hematomas asociados en la placenta y alteraciones anatómicas del útero. Fueron clasificadas como riesgo obstétrico alto $77(63.6 \%)$ y bajo 37 (30.6\%). Según el registro CLAP siete (5.8\%) no tenían riesgo obstétrico.

\section{Tabla 2. Características familiares}

Tipología familiar, frecuencia (\%) †

\begin{tabular}{|l|c|c|}
\hline nuclear & 69 & $(57.0)$ \\
\hline extensa & 25 & $(20.6)$ \\
\hline disuelta & 17 & $(14.0)$ \\
\hline reconstituida & 5 & $(4.1)$ \\
\hline mosaico & 3 & $(2.5)$ \\
\hline vive sola & 1 & $(0.8)$ \\
\hline otra & 1 & $(0.8)$ \\
\hline
\end{tabular}

Ciclo vital familiar, frecuencia (\%) $\ddagger$

\begin{tabular}{|c|c|c|}
\hline expansión & 61 & $(5 \mid .3)$ \\
\hline apertura & 32 & $(26.4)$ \\
\hline consolidación & 28 & $(23.1)$ \\
\hline formación & 0 & $(0)$ \\
\hline posparental & 0 & $(0)$ \\
\hline disolución & 0 & $(0)$ \\
\hline Número de hermanos vivos, promedio (DE) & 2.2 & 1.6 \\
\hline \multicolumn{3}{|l|}{ Apgar familiar (función familiar), frecuencia (\%) } \\
\hline normal $(18-20)$ & 74 & $(6 I .1)$ \\
\hline leve $(14-17)$ & 29 & $(23.9)$ \\
\hline moderada $(10-13)$ & II & $(9.1)$ \\
\hline severa $(<9)$ & 7 & $(5.8)$ \\
\hline \multicolumn{3}{|l|}{ Estado civil padres, frecuencia (\%) } \\
\hline unión libre & 40 & $(33.0)$ \\
\hline separado & 44 & $(36.3)$ \\
\hline casado & 30 & $(24.8)$ \\
\hline viudo & 4 & $(3.3)$ \\
\hline soltero & 3 & $(2.5)$ \\
\hline otro & 0 & $(0)$ \\
\hline \multicolumn{3}{|c|}{ Embarazo adolescente en la familia, frecuencia (\%) } \\
\hline si & 77 & $(63.6)$ \\
\hline no & 44 & $(36.4)$ \\
\hline \multicolumn{3}{|l|}{ Parentesco, frecuencia (\%) } \\
\hline madre & 53 & $(68.8)$ \\
\hline otro familiar & 18 & $(23.4)$ \\
\hline abuela & 5 & $(6.5)$ \\
\hline madre - abuela & 1 & $(1.3)$ \\
\hline Edad, promedio (DE) & 16.8 & $(1.2)$ \\
\hline
\end{tabular}

$\uparrow$ Establece el tipo de familia de origen de acuerdo con el instrumento de medicina familiar, obtenido por interpretación de familiograma; ‡ etapa que se encuentra la familiar de origen. De acuerdo con instrumento de medicina familiar, obtenido por interpretación de familiograma, ciclo definido por el hijo mayor. 
Tabla 3. Características gineco-obstétricas

\begin{tabular}{|c|c|c|}
\hline Edad menarquía, promedio (DE) & 12.7 & $(1.4)$ \\
\hline Edad inicio relaciones, promedio (DE) & 15.6 & $(1.4)$ \\
\hline Planificación familiar, n (\%) & 91 & $(75.2)$ \\
\hline Asistencia programa planificación EPS, n (\%) & 101 & $(83.5)$ \\
\hline Embarazo no deseado, $n$ (\%) & 78 & $(64.5)$ \\
\hline \multicolumn{3}{|l|}{ Número de compañeros sexuales n (\%) } \\
\hline I & 71 & $(58.7)$ \\
\hline 2 a 3 & 41 & $(33.9)$ \\
\hline$>4$ & 9 & (7.4) \\
\hline \multicolumn{3}{|l|}{ Número de embarazos, n (\%) } \\
\hline 1 & 102 & $(84.3)$ \\
\hline 2 & 18 & $(14.9)$ \\
\hline 3 & I & $(0.8 \mathrm{I})$ \\
\hline Abortos, n (\%) & 13 & $(10.7)$ \\
\hline
\end{tabular}

Las comorbilidades durante la gestación se muestran en la Tabla 5. Encontramos un caso de diabetes gestacional, otro de HTA crónica y una preeclampsia, cada uno equivalente al $0.8 \%$. Las patologías maternas más frecuentes fueron infección de vías urinarias en 33 (27.3\%) y vaginosis en 29 (24\%). El estado nutricional evaluado mediante el índice de masa corporal (IMC) reportó normalidad en 74 pacientes $(61.2 \%)$, bajo peso en seis $(4.9 \%)$, sobrepeso en $33(27.3 \%)$ y obesidad en ocho $(6.6 \%)$ (Tabla 5).

En los desenlaces del embarazo encontramos 94 adolescentes $(77.7 \%)$ con parto vaginal y 27 cesáreas $(22.3 \%)$, de los cuales 106 partos fueron a término $(87.6 \%)$ y 13 pretérmino (10.7\%). Se registraron dos casos $(1.6 \%)$ de muerte neonatal producto de gestación de madres en adolescencia tardía, ambas de 19 años, estrato 2, de la localidad de Bosa. Una de ellas con antecedente de hipotiroidismo y otra presentó preeclampsia. Las dos madres terminaron su embarazo por cesárea y estuvieron hospitalizadas.

La complicación perinatal materna más frecuente fue distocia del descenso en once casos $(9.1 \%)$ y las menos frecuentes, distocia de dilatación y hemorragia postparto, cada una con tres casos $(2.5 \%)$.

El peso promedio para los recién nacidos fue 3.122 gramos (DE369) y el bajo peso al nacer fue la compli-
Tabla 4. Seguimiento y curso del embarazo

\begin{tabular}{|c|c|c|}
\hline \multicolumn{3}{|l|}{ Inicio del control prenatal, frecuencia (\%) } \\
\hline primer trimestre & 69 & $(57.0)$ \\
\hline segundo trimestre & 48 & $(39.7)$ \\
\hline tercer trimestre & 4 & (3.3) \\
\hline parto sin controles & 0 & $(0)$ \\
\hline Micronutrientes (ácido fólico), frecuencia (\%) & 117 & $(96.4)$ \\
\hline Embarazo deseado, frecuencia (\%) & 43 & $(35.5)$ \\
\hline Aplicación de vacuna Td, frecuencia (\%) & 93 & $(76.9)$ \\
\hline Valoración odontología, frecuencia (\%) & 85 & $(70.3)$ \\
\hline \multicolumn{3}{|l|}{ Parcial de orina, frecuencia (\%) } \\
\hline normal & 92 & $(76.0)$ \\
\hline patológico & 29 & $(23.9)$ \\
\hline \multicolumn{3}{|l|}{ Glicemia, frecuencia (\%) } \\
\hline normal & 118 & $(97.5)$ \\
\hline patológico $\ddagger$ & 3 & $(2.5)$ \\
\hline \multicolumn{3}{|l|}{ Toxoplasma IgG, frecuencia (\%) } \\
\hline normal & 112 & $(92.6)$ \\
\hline patológico & 9 & (7.4) \\
\hline \multicolumn{3}{|l|}{ Urocultivo, frecuencia (\%) } \\
\hline normal & 93 & (76.9) \\
\hline patológico & 28 & $(23.1)$ \\
\hline \multicolumn{3}{|l|}{ Ecografía, frecuencia (\%) } \\
\hline normal & 112 & $(92.6)$ \\
\hline patológico $¥$ & 9 & (7.4) \\
\hline \multicolumn{3}{|l|}{ Citología } \\
\hline normal & 117 & $(96.7)$ \\
\hline patológico & 4 & (3.3) \\
\hline \multicolumn{3}{|c|}{ Clasificación de riesgo obstétrico, frecuencia (\%) } \\
\hline alto & 77 & $(63.6)$ \\
\hline bajo & 37 & $(30.6)$ \\
\hline ausencia & 7 & (5.8) \\
\hline
\end{tabular}

‡ Glicemia menor de 60 o mayor de $100 \mathrm{mg} / \mathrm{dl}$; ¥cualquier hallazgo en ecografía que pueda afectar el desarrollo normal del embarazo (hematomas, RCIU, alteraciones anatómicas).

cación perinatal más frecuente, en seis casos (4.9\%). La aspiración de meconio, la sepsis neonatal y la macrosomía se presentaron con la misma frecuencia cada una, tres casos (2.5\%), hubo uno de malformación fetal representada por cardiopatía congénita (CIA) (Tabla 6). 
Tabla 5. Comorbilidades durante la gestación

\begin{tabular}{|c|c|c|}
\hline Diabetes gestacional, frecuencia (\%) & 1 & $(0.8)$ \\
\hline IVU, frecuencia (\%) & 33 & $(27.3)$ \\
\hline Vaginosis, frecuencia (\%) & 29 & $(24)$ \\
\hline \multicolumn{3}{|l|}{ Trastorno hipertensivo, frecuencia (\%) } \\
\hline no & 119 & $(98.4)$ \\
\hline HTA crónica & 1 & $(0.8)$ \\
\hline preeclampsia & I & $(0.8)$ \\
\hline HTA gestacional & 0 & (0) \\
\hline eclampsia & 0 & $(0)$ \\
\hline \multicolumn{3}{|l|}{ Estado nutricional, frecuencia (\%) } \\
\hline normal & 74 & $(6 \mid .2)$ \\
\hline sobrepeso & 33 & $(27.3)$ \\
\hline obesidad grado I & 7 & $(5.8)$ \\
\hline bajo peso & 6 & $(4.9)$ \\
\hline obesidad grado II & 1 & $(0.8)$ \\
\hline IMC, promedio (DE) & 24.4 & 3.3 \\
\hline
\end{tabular}

En el análisis de correspondencias múltiples, se encontró que las adolescentes gestantes que presentaron complicaciones clínicas se caracterizaron porque sus embarazos no eran deseados, con antecedentes de embarazos en adolescentes en sus familias, en especial la madre y con disfunción familiar moderada (Figura 1). Las adolescentes embarazadas que tuvieron complicación en el curso de la preñez en mayor proporción provenían de familias extensas (Figura 2).

\section{Discusión}

Las tasas de fecundidad de las adolescentes han descendido en la mayoría de los países en vías de desarrollo durante los últimos veinte años, principalmente debido a la postergación de la primera unión. La fecundidad en Colombia es de cúspide temprana. El grupo de edad con la mayor tasa de fecundidad, 122 nacimientos por mil mujeres, es el de 20 a 24 años, seguido por el de 25 a 29 y el de 15 a 19.

Encontramos en nuestra serie de casos un predominio de embarazos en adolescencia media, de 14 a 17 años, asociados con el inicio de relaciones sexuales en el
Tabla 6. Desenlaces maternos y neonatales

\begin{tabular}{|c|c|c|}
\hline \multicolumn{3}{|l|}{ Desenlace embarazo, frecuencia (\%) } \\
\hline término & 106 & $(87.6)$ \\
\hline pretérmino & 13 & $(10.7)$ \\
\hline mortinato & 2 & $(1.6)$ \\
\hline aborto & 0 & $(0)$ \\
\hline \multicolumn{3}{|l|}{ Vía del parto, frecuencia (\%) } \\
\hline vaginal & 94 & (77.7) \\
\hline cesárea & 27 & $(22.3)$ \\
\hline \multicolumn{3}{|l|}{ Complicaciones materno-fetales } \\
\hline hemorragia postparto, frecuencia (\%) & 3 & $(2.5)$ \\
\hline trastorno hipertensivo, frecuencia (\%) & 5 & $(4.1)$ \\
\hline lesión canal parto, frecuencia (\%) & 5 & $(4.1)$ \\
\hline distocia descenso, frecuencia (\%) & 11 & $(9.1)$ \\
\hline distocia dilatación, frecuencia (\%) & 3 & $(2.5)$ \\
\hline bajo peso, frecuencia (\%) & 6 & $(4.9)$ \\
\hline peso, promedio (DE) & 3122 & 369.7 \\
\hline apiración de meconio, frecuencia (\%) & 3 & $(2.5)$ \\
\hline sepsis neonatal, frecuencia (\%) & 3 & $(2.5)$ \\
\hline macrosomia, frecuencia (\%) & 3 & $(2.5)$ \\
\hline malformación, frecuencia (\%) & 1 & $(0.8)$ \\
\hline \multicolumn{3}{|l|}{ Destino de la madre, frecuencia (\%) } \\
\hline domicilio & 104 & $(85.9)$ \\
\hline hospitalización & 17 & $(14.1)$ \\
\hline $\mathrm{UCl}$ & 0 & $(0)$ \\
\hline \multicolumn{3}{|l|}{ Destino del producto, frecuencia (\%) } \\
\hline domicilio & 101 & $(83.5)$ \\
\hline hospitalización & 18 & $(14.9)$ \\
\hline $\mathrm{UCl}$ & 2 & $(1.69)$ \\
\hline
\end{tabular}

mismo grupo de edad, se evidenció además que la edad mínima fue a los 14 años, con un promedio de las gestantes de 17.3 años. De igual manera crece cada vez más el número de casos en población de estratos socioeconómicos bajos, con mayor concentración hacia el suroccidente de la ciudad, en especial Bosa, Kennedy, Ciudad Bolívar y San Cristóbal, resultados concordantes con lo descrito en la Dirección de Censos y Demografía en 2010.

La ocupación muestra en forma llamativa un gran número de adolescentes dedicadas a actividades del hogar asociada con deserción escolar, la mayoría cursando bachillerato o con estudios hasta este nivel, 


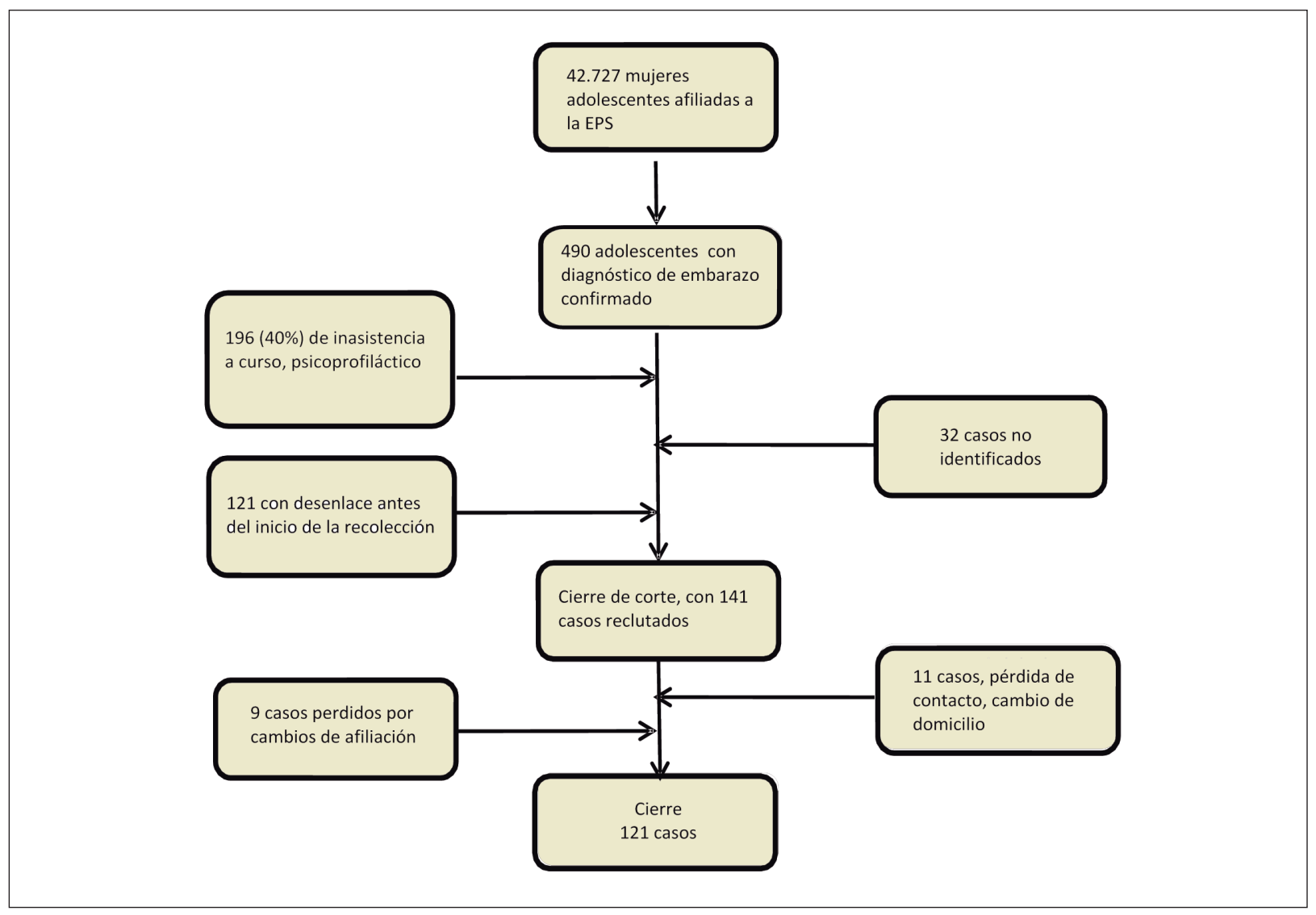

Figura I. Selección de pacientes.

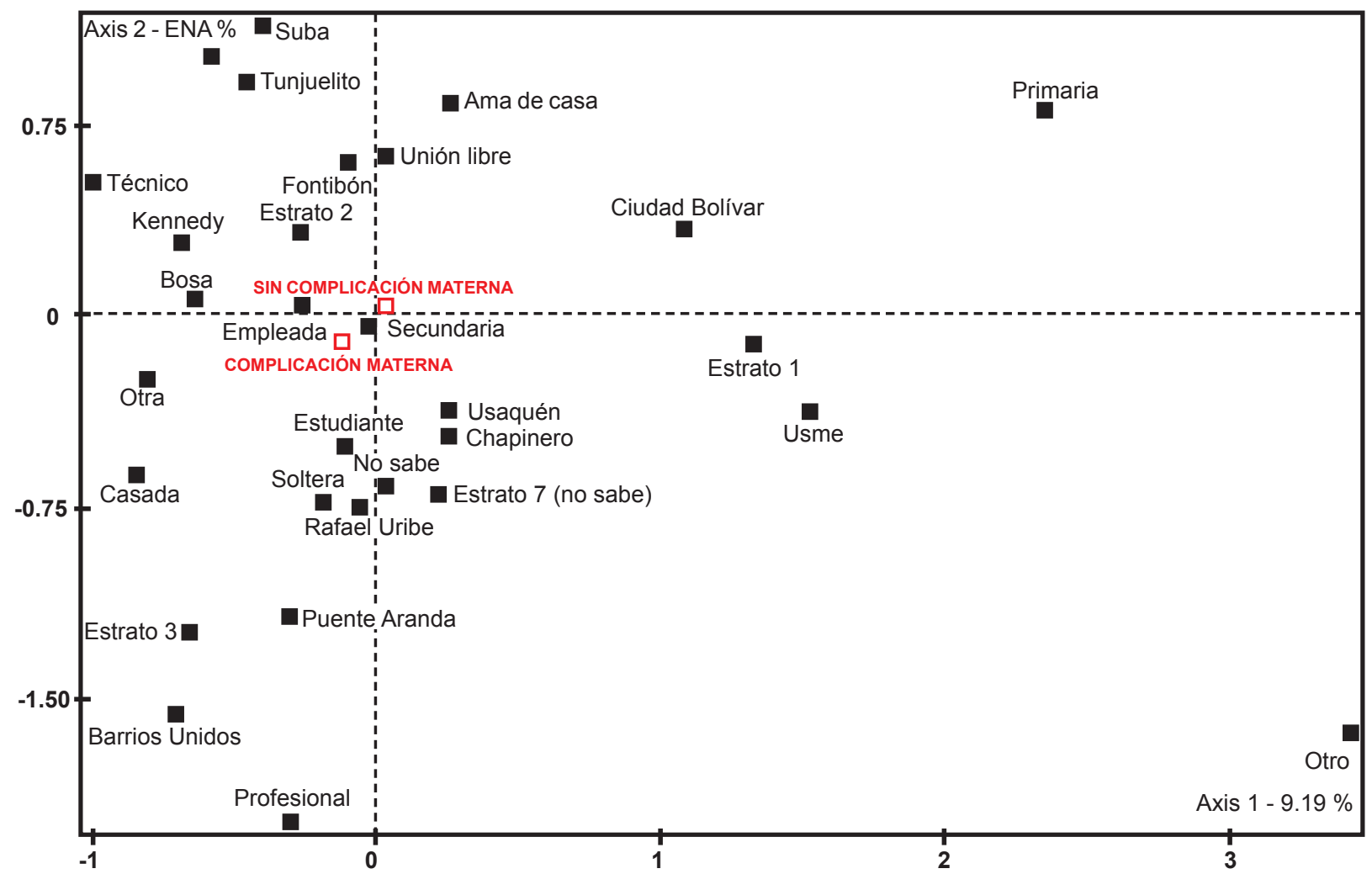

Figura 2. Características demográficas y complicaciones maternas. 
que podría ser una consecuencia de su embarazo; un poco más de la mitad llevaban vida de pareja, la mayoría en unión libre, asumiendo su rol de esposas lo que cambia las funciones de su ciclo vital individual, esperando que sea un período definitivo en la adquisición de conocimientos y valores para definir su rol familiar y social, mediante la identidad del yo, tienen que asumir las tareas derivadas de la conformación de los nuevos núcleos familiares, no propias para su edad.

En cuanto a las características familiares, las cuales hasta el momento no se han descrito en otras series evaluando la tipología familiar ni la funcionalidad asociada con la gestación, curso y desenlace, además de las condiciones clínicas encontramos un cambio importante en la tipología familiar esperada para el grupo poblacional por su edad, con aumento importante de la familia nuclear, lo cual se explica por el mismo incremento comentado en pacientes viviendo en unión libre y con familia extensa. Es una de las variables importantes para complicaciones tanto maternas como fetales y desenlaces, afectando también el ciclo vital familiar con predominio de familias en expansión y en apertura determinadas por el embarazo evaluado y la misma adolescente. Es importante anotar cómo desde la medicina familiar se observa una asociación entre el cambio del ciclo vital individual de adolescente a adulto joven, con la independencia de su familia de origen y la decisión de conformar una nueva, con todas las herramientas para ser el nuevo líder de hogar para lo cual las adolescentes no están preparadas.

Para la funcionalidad familiar se encuentra una frecuencia baja con disfunción severa, sin que haya asociación con complicaciones maternas, fetales ni el curso del embarazo, a diferencia de la disfunción moderada que se relacionó con complicaciones maternas. Ante la disfunción asociada con los cambios descritos de conformación de familias con núcleo adolescente, convendría proponer nuevos estudios donde se pueda evaluar la asociación directa de las complicaciones con la tipología y funcionalidad familiar.

Es importante destacar la posible relación causal de antecedente familiar de embarazo en adolescente y los nuevos casos, descritos en series internacionales y vista en nuestro estudio con el $63.6 \%$, con mayor frecuencia cuando la madre tiene edad promedio menor a la de la serie evaluada. Es de anotar la limitación aparente del estudio al ser realizado en una población urbana pues el mayor antecedente de familiar de adolescente gestante se encuentra descrito en otras series en población rural. ${ }^{17}$

La encuesta nacional de demografía y salud Colombia 2010 evaluó 49.818 mujeres en edad fértil entre los 15 y 49 años y el $99.9 \%$ conocía algún método anticonceptivo de los descritos en la encuesta. El estudio de Nocaima (Cundinamarca) en adolescentes de 14 a 19 años, estableció el conocimiento que tienen las adolescentes sobre los métodos de prevención del embarazo. Sin embargo, llama la atención que en nuestro estudio a pesar de su bajo deseo del embarazo, no planificaban ni asistían a un programa sobre el tema, asumiendo conductas de riesgo que las llevaron a esa situación. ${ }^{17-23}$

Los resultados de los estudios de tamizaje y seguimiento del embarazo no muestran ninguna alteración predominante dentro de la caracterización realizada, con frecuencias de alteraciones esperadas y de igual comportamiento para las series descritas en otros estudios, con predominio de infección de vías urinarias y vaginosis, lo que aumenta el riesgo de complicaciones durante la gestación independiente de la edad de la embarazada.

Al analizar las variables determinadas para embarazo actual, encontramos puntos definitivos que pueden generar hipótesis importantes para el manejo de nuestra población de interés; la mayoría manifiesta que su embarazo fue deseado en proporción relativa al mismo número de casos que tiene pareja estable y no acuden a los controles prenatales de manera temprana a la valoración odontológica, la vacunación y menos a la ingesta de micronutrientes. Lo anterior pareciera contradictorio, pero entendible ante la edad y características cognitivas descritas para nuestro grupo estudiado, por lo cual la mayoría se clasifican con embarazo de alto riesgo asociado con inicio tardío de seguimiento.

En los desenlaces de la gestación se encontró un mayor porcentaje de complicaciones maternas solo para 
las distocias de dilatación y del descenso, comparado con grupos de mujeres no adolescentes. Las demás complicaciones maternas y fetales no revelaron características particulares que difieran con mujeres mayores de veinte años. Se realizó una descripción de las características evaluadas, distribuyendo las adolescentes por subgrupos en medias ( $<17$ años) y finales (>17 años) sin encontrar diferencias en la frecuencia de complicaciones durante la gestación.

La mortalidad perinatal encontrada en el estudio fue de $1.6 \%$, cercana a la reportada en otras $\operatorname{series}^{1-3}$ para mujeres con características similares. Tampoco se encuentra diferencia con la estadística de la ENDS 2010, con mortalidad neonatal en Bogotá de 1.7\% y nacional $1.2 \% .^{23}$

Al realizar el análisis con las variables ilustrativas respecto a complicaciones maternas en el desenlace de la gestación, o las que aparecen en el curso de la gestación o las fetales en el desenlace de la gestación, al relacionarlas con variables demográficas se encontró en todas la series mayor frecuencia para la ocupación, el estado civil soltera y la localidad en la ciudad. Para las variables clínicas no se observaron cambios a los ya descritos en la literatura para la edad de menarquia e inicio de relaciones sexuales tempranas, sin ninguna predominancia en los estudios complementarios. En las variables familiares se vio correspondencia en grupos con familias extensas, asociación con disfunción familiar moderada y antecedente de gestación en adolescentes en la familia.

Consideramos los resultados importantes pues permiten identificar puntos clave de intervención temprana en la IPS evaluada, no solo en la atención de gestantes adolescentes sino en aquellas con vida sexual activa, permitiendo controlar no solo el aumento exponencial de nuevos embarazos en este grupo poblacional, sino también preparando y educando para la maternidad segura y la adquisición de capacidades para ejercer funciones, sin estar preparadas en su ciclo vital individual.

Se debe considerar la posibilidad de evaluar características predominantes encontradas para la descripción familiar, hasta el momento no estudiadas: ¿Existirá relación directa entre la disfunción familiar moderada y severa con el deseo de embarazo y la formación de nuevos núcleos familiares? ¿La formación de núcleos familiares en adolescentes perpetúa las crisis familiares y la cadena de embarazos a temprana edad? ¿Existe relación directa entre los grupos de adolescentes y la aparición de complicaciones? Se debe determinar a partir de esta caracterización los puntos de trabajo que no están mostrando resultados: ¿El programa de planificación familiar, no solo de la IPS sino de nuestro sistema, es el adecuado para la población de adolescentes? ¿La educación sexual no está siendo percibida con la finalidad que fue diseñada?

Consideramos como limitación importante del estudio y factor de riesgo para desenlaces no esperados la ausencia de población en adolescencia temprana, lo cual reduce la frecuencia de complicaciones, así como la alta inasistencia a controles prenatales y cursos de preparación para la maternidad, lo que limita momentos cruciales de atención y prevención primaria. Esperamos generar nuevas hipótesis con el conocimiento de las características evaluadas en nuestra población de adolescentes gestantes.

\section{Conclusión}

La serie descrita muestra características demográficas que sugieren problemas importantes en educación y control de anticoncepción, que deben tenerse en cuenta para nuevos estudios y planteamiento de acciones de mejora del programa de atención a adolescentes y anticoncepción en la institución evaluada. Se encuentra entre las adolescentes embarazadas un predominio de familias nucleares y extensas, asociadas con disfunción familiar importante en cerca de la mitad de los casos, que debe evaluarse para acciones a nivel de comunidad y servicios de atención integral como medicina familiar. Aunque no se evidencia mayor prevalencia de complicaciones en el curso y desenlace de gestación al compararla con otras series o grupos poblacionales, no se puede desconocer el riesgo social derivado de la disfunción familiarcon factores de potencial intervención. No se encontró ninguna variable clínica como factor predominante para las 


\section{complicaciones evaluadas. La mortalidad neonatal es} igual a la observada para todas las edades a nivel local y nacional, asociada con comorbilidad de base que permanece dentro de la estadística descrita.

\section{Referencias}

1. Smith GC, Pell JP. Teenage pregnancy and risk of adverse perinatal outcomes associated with first and second births: population based retrospective cohort study. BMJ 2001 Sep 1;323(7311):476.

2. Jorge Peláez Mendoza. Adolescente embarazada: características y riesgos. Rev CubanaObstetGinecol 1997 Oct 10;23(1):13-7.

3. Conde-Agudelo A, Belizan JM, Lammers C. Maternal-perinatal morbidity and mortality associated with adolescent pregnancy in Latin America: Cross-sectional study. Am J ObstetGynecol 2005 Feb;192(2):342-9.

4. Stevens-Simon C, Beach RK, McGregor JA. Does incomplete growth and development predispose teenagers to preterm delivery? A templateforresearch. J Perinatol 2002 Jun;22(4):315-23.

5. Becerra D, Becerra D, Parra Pineda MO. Embarazo en la Adolescencia. Universidad Nacional de Colombia. Universidad Nacional de Colombia.; 2009.

6. Cabrero R CR. La gestante adolescente. In: Editorial Medica Panamericana, editor. Obstetricia y medicina materno-fetal. 2007.

7. Dirección de Censos y Demografía - Estadísticas vitales; 2009

8. Ministerio de la Protección Social PIUU. Encuesta Nacional de Demografía y Salud. Bogotá. 2005

9. Florez CE, Vargas E, Henao J, Gonzàles C, Soto V, Kassem D. Fecundidad en Adolescentes en Colombia: Incidencia, tendencias y determinantes. Un enfoque de Historia de vida. Universidad de los Andes, Bogotá Agosto 2004.

10. Tovar M, Burbano C, Mora M, Domínguez M, García A. Estudio comparativo de embarazadas adolescentes versus embarazadas adultas jóvenes en el Centro Hospital Primitivo Iglesias de la ciudad de Cali. Rev.colomb.obstet.ginecol 38[6], 436-445. 21987. RefType: Magazine Article
11. Cáceres J, Escudero Carranza V. Relación de pareja en jóvenes y embarazos no deseados. 1998.

12. Chalem F, Nieto MC, Casasbuenas J, Ahumada J, Esguerra R, Chalem P. ECMG, Medicina Familiar, Educación continuada para el médico general. ECMG 2002 Jan $1 ;(4)$.

13. Terrasa S, Rubinstein A. La Salud del Adolescente. In: Editorial Médica Panamericana S.A., editor. Medicina Familiar y Práctica Ambulatoria. 2 ed. Buenos Aires: 2006

14. Tapia C, Pérez P, Gutiérrez M, Villaseñor M, Gutiérrez Z. Embarazo en la adolescencia. Aspectos obstétricos, perinatales, psicológicos y socioculturales. In: El Manual Moderno, editor. La salud del niño y el adolescente. 5 ed. México: 2005. p. 1621-7.

15. Amaya J, Borrero C, Ucrós S. Estudio analítico del Resultado del embarazo en adolescentes y mujeres de 20 a 29 años en Bogotá. Revista Colombiana de Obstetricia y Ginecología 2005 Sep 26;56(3):216-24.

16. Ministerio de la Protección Social - MPS. Guía para la detección temprana de las alteraciones del embarazo. 2007 Junio 19.

17. Alvarado Ricardo, Martínez Pachón Oscar. Problemática entorno al embarazo en adolescentes de 14 a19 años, en Nocaima (Cundinamarca). Revista Colombiana de ciencia Salud. Bogotá (Colombia) 5 (1): 40 - 5 2, abril - junio de 2007

18. Loeche, M. Martin, Catalá E, Ortiz R.M, Análisis epidemiológico de los resultados perinatales obtenidos en las gestantes adolescentes de la Comunidad Valenciana. ProgObstetGinecol 2003; 46(2):53-8.

19. Langerana, El embarazo no deseado: impacto sobre la salud y la sociedad en América Latina y el Caribe Revista Panameña Salud Publica/Pan Am J PublicHealth 11(3), 2002193.

20. Dorta E, Molina J, García Fernández J.A., Gestación adolescente y su repercusión en el recién nacido. Estudio de casos y controles, clínica investigación ginecología y Obstetricia 2004; 31(4):118-23

21. Abril Valdés Stevens Complicaciones del embarazo en adolescentes gestantes UPSS Santa Cruz 2008-2009. Trabajo de grado para obtener Título de médico.

22. Rubinstein R. Medicina Familiar y Práctica Ambulatoria, 2da. Ed. Editorial Médica Panamericana, Buenos Aires, 2006; 8(73-74)599-621.

23. Ministerio de la Protección Social PIUU. Encuesta Nacional de Demografía y Salud. Bogotá. 2010 